\title{
Finite Difference Approximation of Hedging Quantities in the Heston model
}

\author{
Karel in 't Hout \\ Department of Mathematics and Computer Science, University of Antwerp, \\ Middelheimlaan 1, 2020 Antwerp, Belgium
}

\begin{abstract}
This note concerns the hedging quantities Delta and Gamma in the Heston model for European-style financial options. A modification of the discretization technique from In 't Hout \& Foulon (2010) is proposed, which enables a fast and accurate approximation of these important quantities. Numerical experiments are given that illustrate the performance.
\end{abstract}

Keywords: financial option pricing, hedging quantities, Heston model, finite difference methods.

PACS: $02.60 . \mathrm{Lj}, 02.70 . \mathrm{Bf}$, 89.65.Gh

\section{HESTON MODEL AND HEDGING QUANTITIES}

The Heston partial differential equation (PDE) for the valuation of European-style financial options is given by

$$
\frac{\partial u}{\partial t}=\frac{1}{2} s^{2} v \frac{\partial^{2} u}{\partial s^{2}}+\rho \sigma s v \frac{\partial^{2} u}{\partial s \partial v}+\frac{1}{2} \sigma^{2} v \frac{\partial^{2} u}{\partial v^{2}}+r s \frac{\partial u}{\partial s}+\kappa(\eta-v) \frac{\partial u}{\partial v}-r u \quad(s>0, v>0,0<t \leq T) .
$$

Here $u(s, v, t)$ denotes the fair value of the option if at time $T-t$ the underlying asset price equals $s$ and its variance equals $v$ with $T$ the given maturity time of the option. The parameter $\kappa>0$ is the mean-reversion rate, $\eta>0$ is the long-term mean, $\sigma>0$ is the volatility-of-variance, $\rho \in[-1,1]$ is the correlation between the two underlying Brownian motions and $r$ denotes the risk-neutral interest rate. The Heston PDE (1) can be regarded as a time-dependent convection-diffusion-reaction equation on an unbounded two-dimensional spatial domain. It constitutes the keystone of many contemporary models in foreign exchange (FX) option markets. Besides the actual option value, also the hedging quantities, often called the Greeks, are of prime importance. In particular, the Delta $\Delta$ and the Gamma $\Gamma$ of an option are defined by

$$
\Delta=\frac{\partial u}{\partial s} \quad \text { and } \quad \Gamma=\frac{\partial^{2} u}{\partial s^{2}} .
$$

Clearly $\Delta$ represents the rate of change of the option value with respect to the underlying asset price $s$ and $\Gamma$ represents the rate of change of $\Delta$ with respect to $s$. These quantities are crucial when hedging an option. For most types of options, analytic formulas in (semi-)closed form are not known in the literature for either their fair values or their hedging quantities. Accordingly, one resorts to numerical techniques for their approximation. In [4] the numerical solution of the Heston PDE was considered following the well-known method-of-lines approach. Here the PDE has been semidiscretized in the $(s, v)$-domain by applying finite difference (FD) schemes on nonuniform Cartesian grids. For the effective numerical solution of the resulting large systems of stiff ordinary differential equations (ODEs), various Alternating Direction Implicit (ADI) splitting schemes have been investigated. The attention in [4] was directed towards approximating option values $u$ itself. In principle, the ADI FD approach can directly be used for approximating hedging quantities such as $\Delta$ and $\Gamma$ as well. However, the initial function for (1), which is given by the payoff of the pertinent option, is in general nonsmooth. This leads to difficulties in the numerical approximation, which are more severe for the hedging quantities than for the option value itself. In the present note we formulate a modification of the numerical technique from [4], with the purpose to efficiently approximate also $\Delta$ and $\Gamma$. This modification concerns the FD discretization. We shall consider here the prototype case of European call options. Then the initial condition is

$$
u(s, v, 0)=\max (0, s-K) \quad(s \geq 0, v \geq 0),
$$

where $K>0$ denotes the given strike price of the option. Clearly, the initial function is nonsmooth in $s=K$. As boundary condition in $s=0$ there holds

$$
u(0, v, t)=0 \quad(v \geq 0,0<t \leq T) .
$$


The boundary $v=0$ is special in that all second-order derivative terms in the Heston PDE (1) vanish as $v \downarrow 0$. It has recently been proved in [2] that inserting $v=0$ into (1) actually gives the correct condition there. We note that this is irrespective of whether or not the well-known Feller condition $2 \kappa \eta>\sigma^{2}$ holds.

\section{FINITE DIFFERENCE DISCRETIZATION}

For the semidiscretization of the Heston PDE the $(s, v)$-domain is first restricted to a bounded set $\left[0, S_{\max }\right] \times\left[0, V_{\max }\right]$ with fixed values $S_{\max }, V_{\max }$ chosen sufficiently large. In view of the option and model parameters in the numerical example presented below, we have (empirically) chosen $S_{\max }=14 K, V_{\max }=10$. The following boundary conditions are imposed,

$$
\frac{\partial u}{\partial s}\left(S_{\max }, v, t\right)=1 \quad(v \geq 0,0<t \leq T) \quad \text { and } \quad u\left(s, V_{\max }, t\right)=s \quad(s \geq 0,0<t \leq T) .
$$

Let integers $m_{1}, m_{2} \geq 1$ be given and arbitrary. The initial-boundary value problem (1)-(4) is semidiscretized on a Cartesian grid $\left\{\left(s_{i}, v_{j}\right): 1 \leq i \leq m_{1}, 0 \leq j \leq m_{2}-1\right\}$ with smooth, nonuniform meshes $0=s_{0}<s_{1}<\ldots<s_{m_{1}}=S_{\max }$ and $0=v_{0}<v_{1}<\ldots<v_{m_{2}}=V_{\max }$ in the $s$ - and $v$-directions as defined in [3]. For brevity we omit the precise formulas, but mention that in the $s$-direction relatively many mesh points are placed in a suitable interval containing the strike $K$ and in the $v$-direction relatively many mesh points are placed near the boundary $v=0$. In practice this concerns the region of interest and the use of this nonuniform grid greatly improves the accuracy of the FD discretizion compared to employing a uniform grid. The actual FD discretization is performed using second-order schemes as described in detail in [4], with one modification. Instead of taking the second-order central scheme for the $\partial u / \partial s$ term in (1) we choose here the second-order upwind scheme. The reason is that in approximating Delta and Gamma the central scheme can give rise to spurious oscillations that are alleviated when applying the upwind scheme. A last modification to enable efficient approximation of Delta and Gamma is to replace the value of the payoff function (2) at the grid point $s_{i}$ nearest to the strike $K$ by its cell average,

$$
\frac{1}{h} \int_{s_{i-1 / 2}}^{s_{i+1 / 2}} \max (0, s-K) d s \quad \text { with } s_{i-1 / 2}=\frac{1}{2}\left(s_{i-1}+s_{i}\right), s_{i+1 / 2}=\frac{1}{2}\left(s_{i}+s_{i+1}\right), h=s_{i+1 / 2}-s_{i-1 / 2} .
$$

This reduces the dependency of the discretization error on the location of the strike relative to the $s$-mesh, see e.g. [7].

The semidiscretization outlined above leads to initial value problems for large systems of ODEs. For their numerical solution, ADI schemes form an effective means. Here we choose the Modified Craig-Sneyd (MCS) scheme, with parameter $\theta=\frac{1}{3}$, see e.g. [4].

Clearly the above yields approximations $U_{i j}(t) \approx u\left(s_{i}, v_{j}, t\right)$ of the option values at the grid points. Since Delta and Gamma appear themselves as terms in the Heston PDE, a natural and direct way to approximate these quantities is to apply the two pertinent FD schemes in the $s$-direction to $U_{i j}(t)$. This concerns two simple matrix multiplications. In the following we shall investigate by numerical experiment the accuracy of these approximations. This is interesting, notably, considering the nonsmoothness of the initial function at $s=K$. We study the maximum error at time $T$ over a region of interest in $(s, v)$-space given here by $\left(\frac{1}{2} K, \frac{3}{2} K\right) \times(0,1)$. The MCS scheme is applied with sufficiently small time step so that the temporal error is negligible. For the exact values of Delta and Gamma we employ the semi-closed form analytic formula as given e.g. in [6].

\section{NUMERICAL EXPERIMENTS}

For the numerical example we take a test case from [1] for the Heston model, where the Feller condition is not satisfied:

$$
\kappa=1, \eta=0.09, \sigma=1, \rho=-0.3, \quad r=0, T=5, \quad K=100 .
$$

The left-hand side of Figure 1 displays, from top to bottom, the corresponding option value function $u$, the Delta function and the Gamma function on $[0,2 K] \times[0,1]$ obtained with the above discretization method using $m_{1}=100$, $m_{2}=50$. The right-hand side of Figure 1 shows the pertinent discretization errors where $m_{1}=2 m, m_{2}=m$ with $m=10,20, \ldots, 100$. An examination of the outcomes indicates a convergence order equal to two, for both the option value and Delta and Gamma, which is clearly a favorable result. We mention that similar positive results were found 

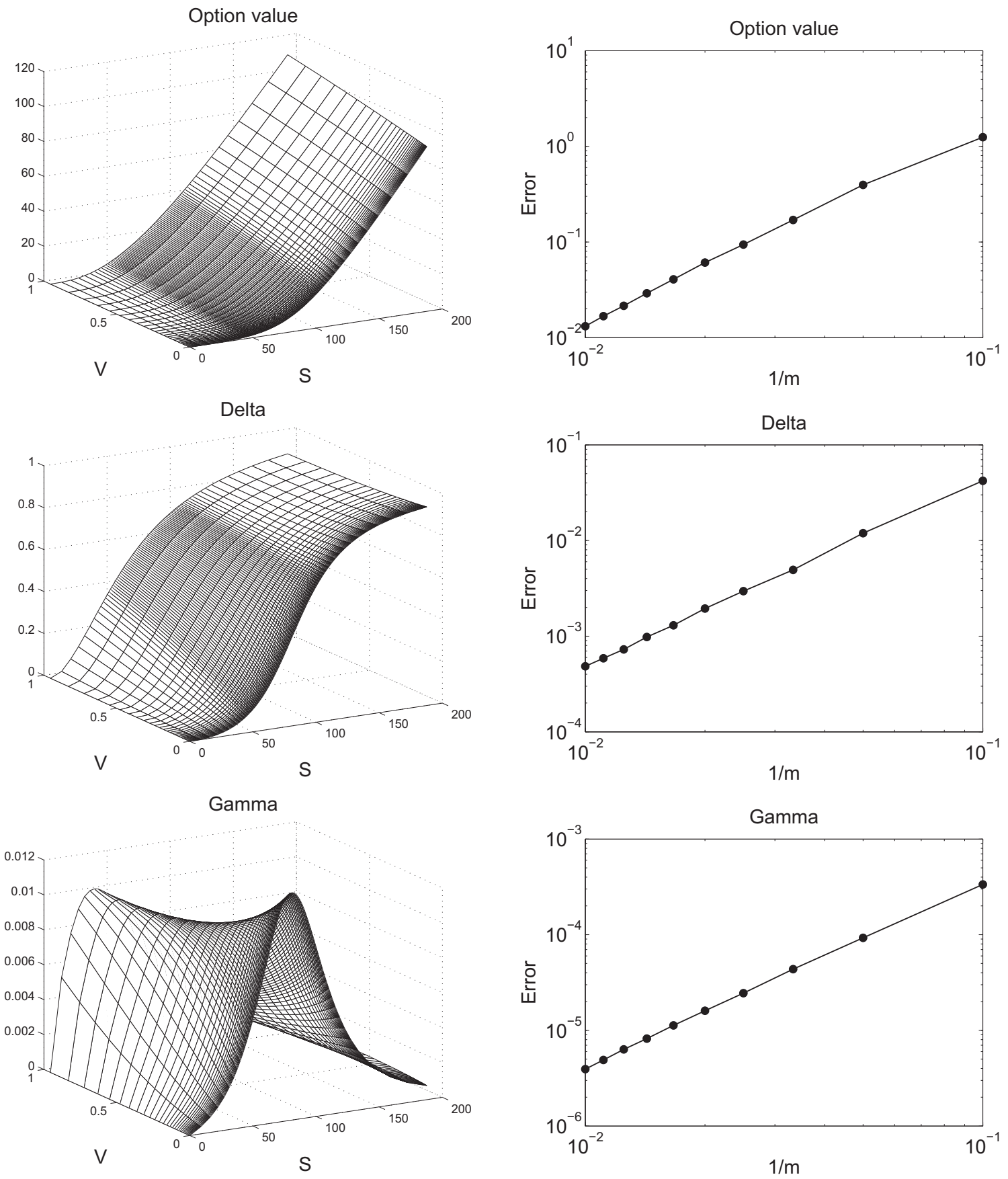

FIGURE 1.

in all other (six) test cases from [1, 4]. In future research we shall consider also other hedging quantities and exotic options for which analytic formulas in semi-closed form are not known under the Heston model, or extensions thereof. Finally, advanced methods for avoiding spurious oscillations, such as TVD or ENO techniques, may be important when the maturity time $T$ is small. Such techniques have been considered in financial option pricing e.g. in $[5,8]$. 


\section{ACKNOWLEDGEMENTS}

I am grateful to Peter Forsyth for pointing to the reference by Ekström \& Tysk (2010). Also I would like to thank Tinne Haentjens for useful discussions on the topic of the present note. This work has been supported financially by the Research Foundation - Flanders, FWO contract no. G.0125.08.

\section{REFERENCES}

1. L. Andersen, Simple and efficient simulation of the Heston stochastic volatility model, J. Comp. Finan. 11 (2008) 1-42.

2. E. Ekström \& J. Tysk, The Black-Scholes equation in stochastic volatility models, J. Math. Anal. Appl. 368 (2010) $498-507$.

3. T. Haentjens \& K. J. in 't Hout, Alternating direction implicit finite difference schemes for the Heston-Hull-White partial differential equation, J. Comp. Finan. (2012), to appear.

4. K. J. in 't Hout \& S. Foulon, ADI finite difference schemes for option pricing in the Heston model with correlation, Int. J. Numer. Anal. Mod. 7 (2010) 303-320.

5. C. W. Oosterlee, J. C. Frisch \& F. J. Gaspar, TVD, WENO and blended BDF discretizations for Asian options, Comp. Visual. Sc. 6 (2004) 131-138.

6. O. Reiss \& U. Wystup, Computing option price sensitivities using homogeneity, J. Deriv. 9 (2001) 41-53.

7. D. Tavella \& C. Randall, Pricing Financial Instruments, Wiley, New York, 2000.

8. R. Zvan, P. A. Forsyth \& K. R. Vetzal, Robust numerical methods for PDE models of Asian options, J. Comp. Finan. 1 (1998) 39-78. 
Copyright of AIP Conference Proceedings is the property of American Institute of Physics and its content may not be copied or emailed to multiple sites or posted to a listserv without the copyright holder's express written permission. However, users may print, download, or email articles for individual use. 